\title{
Application of compost in mixed media improved oil palm nursery's secondary root structure thereby reducing the fertilizer requirement for growth
}

\author{
Siti Suliza Salamat ${ }^{\mathrm{a}, \mathrm{b}}$, Mohd Ali Hassan ${ }^{\mathrm{a}^{*}}$, Yoshihito Shirai ${ }^{\mathrm{b}}$, Ahmad Husni Mohd Hanif ${ }^{\mathrm{c}}$, Izwanizam \\ Arifin $^{\mathrm{d}}$, Mohamad Shahkhirat Norizan ${ }^{\mathrm{d}}$ \\ ${ }^{a}$ Department of Bioprocess Technology, Faculty of Biotechnology and Biomolecular Science, Universiti Putra Malaysia, 43400 UPM Serdang, \\ Selangor, Malaysia \\ ${ }^{b}$ Department of Biological Functions and Engineering, Graduate School of Life Science and Systems Engineering, Kyushu Institute of Technology, \\ 2-4 Hibikino, Wakamatsu-ku, Kitakyushu, Fukuoka 808-0196, Japan \\ 'Department of Soil Science, Faculty of Agriculture Universiti Putra Malaysia, 43400 UPM Serdang, Selangor, Malaysia \\ ${ }^{d U n i t ~ A g r o n o m i ~ S a w i t, ~ P u s a t ~ P e n y e l i d i k a n ~ P e r t a n i a n T u n ~ R a z a k, ~ F E L D A ~ A g r i c u l t u r a l ~ S e r v i c e s ~ S d n ~ B b d, ~} 26400$ Bandar Tun Abdul \\ Razak Jengka, Pahang, Malaysia
}

Received 13th November 2018 / Accepted 19th June 2019

\begin{abstract}
Although conventional inorganic fertilizers increased plant growth and productivity, their excessive use leads to wastage, run-offs and environmental pollution. In order to promote nutrient recycling and zero emission strategy in the palm oil industry, compost was produced from oil palm empty fruit bunch (EFB) and palm oil mill effluent (POME) anaerobic sludge. The main objective of this study is to determine the effect of compost in the mixed media on the growth and inorganic fertilizer requirement in the oil palm nursery. $100 \%$ soil as media with 100\% inorganic fertilizer as control was compared with mixed media containing 50\% compost in soil with inorganic fertilizer at $25 \%, 50 \%, 75 \%$ and 100\% compositions and tested for plant growth and root structure. The results showed that the treatments with compost addition improved plant growth, compared to the control with $100 \%$ inorganic fertilizer which did not contain compost in the mixed medium. The improved plant growth corresponded directly to the enhanced secondary root structure, which probably resulted in more efficient absorption and uptake of nutrients by the plants. Furthermore, the plant growth and the secondary root structure in the mixed media with 50\% inorganic fertilizer composition was not significantly different to the media with $75 \%$ and $100 \%$ inorganic fertilizer. Therefore it is suggested that the application of $50 \%$ compost in the mixed media enhanced the secondary root structure, resulting in reduced inorganic fertilizer requirement in the oil palm main nursery, without affecting the plant growth.
\end{abstract}

Keywords: compost, planting media, oil palm nursery

\section{INTRODUCTION}

Oil palm (Elaeis guineesis) is an important commercial agriculture crop which provides income and employment in the agricultural sector in the tropics. The waste and resources from the non-oil streams in this industry are rich in nutrients which can generate additional economic value. The empty fruit bunch (EFB) and palm oil mill effluent (POME) anaerobic sludge are two

* Author for correspondence: Mohd Ali Hassan, Department of Bioprocess Technology, Faculty of Biotechnology and Biomolecular Sciences, Universiti Putra Malaysia, 43400 UPM Serdang, Selangor, Malaysia. Email alihas@upm.edu.my 
raw materials that can be converted to organic fertilizer, which can improve soil fertility and conditions the soil in oil palm plantations. The prolonged cultivation of oil palm depleted the essential nutrients and soil cation exchange capacity (CEC). This can be alleviated by the application of organic fertilizer from EFB and POME anaerobic sludge (Ashraf et al., 2017), which is an excellent nutrient-recycling strategy within the industry. The palm oil industry conventionally used topsoil in the media for the oil palm main nursery. Due to the limitation of good topsoil, application of inorganic fertilizers has been widely used which contributed to soil acidification, declined $\mathrm{pH}$ and low fertility of soil as media for oil palm nursery (Rosenani et al., 2016). An alternative growth medium is desirable to produce high quality seedlings, particularly by enhancing plant growth and root structure. It has been proposed that EFB and POME anaerobic sludge can improve soil fertility by increasing the water holding capacity, total nitrogen content, available phosphorus content, CEC and microbial activity (Ramli et al., 2016). In a previous study by Siregar et al. (2011), the growth in the main nursery with mixed media comprising of top soil and EFB compost was similar to topsoil with the normal fertilizer application. Rosenani et al. (2016) reported that the use of EFB in seedling planting nursery improved soil chemical properties and vegetative growth. In addition, Albregts and Chandler (1993) reported that the level and form of the fertilizer applied affected the root and shoot morphology. The aim of this study is to determine the effect of compost from EFB and POME anaerobic sludge as a component in the mixed media on the growth and inorganic fertilizer requirement of oil palm main nursery plants.

\section{MATERIALS AND METHODS}

Treatments. The study was conducted in Ladang 10, Universiti Putra Malaysia with daily temperature variation of $24-33^{\circ} \mathrm{C}$. Pressedshredded $\mathrm{EFB}$ and POME anaerobic sludge obtained from Seri Hulu Langat palm Oil Mill and FELDA Trolak Palm Oil Mill, respectively, were composted at the Biorefinery Complex, Universiti
Putra Malaysia as reported by (Baharuddin et al., 2009). Oil palm seeds, Dura x Psifera, were obtained from FELDA Agricultural Services Sdn. Bhd, Pusat Penyelidikan Tun Razak, Bandar Jengka, Pahang, Malaysia. Five polybag media were prepared with $100 \%$ Serdang series topsoil and compost (50\% topsoil: $50 \%$ compost $\mathrm{v} / \mathrm{v}$ ) with different percentages of inorganic fertilizer, as shown in Table 1. The experiment was carried out in a randomized complete block design (RCBD) with four replications. The planting distance between each polybag in the block was 3 ft. x $3 \mathrm{ft}$. (triangle). One oil palm seed was sown in each polybag of $38 \mathrm{~cm} \mathrm{x} 45 \mathrm{~cm}$ with equal volume per volume of media. The seedlings were watered twice a day by using the drip system and weeded manually. An inorganic fertilizer, NPK Yellow (15:15:6) was applied from week 1 (10 $\mathrm{g} /$ plant), week 2 (15 g/plant), weeks 3-5 (20 $\mathrm{g} /$ plant $)$ and weeks $6-8$ (30 g/plant), as used in conventional agricultural practise at the oil palm main nursery. Composite sample was taken from 10 seedlings per replication and 4 replications for every sampling. Table 1 showed treatment on this experiment, i.e. $100 \%$ soil with $100 \%$ inorganic fertilizer (Treatment 1) as control, $50 \%$ soil: $50 \%$ compost with $100 \%$ inorganic fertilizer (Treatment 2), 50\% soil: 50\% compost with $100 \%$ inorganic fertilizer (Treatment 3), 50\% soil: $50 \%$ compost with 50\% inorganic fertilizer (Treatment 4) and 50\% soil: 50\% compost with 25\% inorganic fertilizer (Treatment 5).

Table 1. List of treatment used to treat oil palm seedling in this study.

\begin{tabular}{|c|c|}
\hline Treatment & $\begin{array}{c}\text { Percentage soil with inorganic } \\
\text { fertilizer }\end{array}$ \\
\hline T1 & $\begin{array}{l}100 \% \text { Soil With } 100 \% \text { Inorganic } \\
\text { fertilizer }\end{array}$ \\
\hline $\mathrm{T} 2$ & $\begin{array}{l}50 \% \text { Soil : } 50 \% \text { Compost With } 100 \% \\
\text { Inorganic fertilizer }\end{array}$ \\
\hline T3 & $\begin{array}{l}50 \% \text { Soil : } 50 \% \text { Compost With } 75 \% \\
\text { Inorganic fertilizer }\end{array}$ \\
\hline T4 & $\begin{array}{l}50 \% \text { Soil : } 50 \% \text { Compost With } 50 \% \\
\text { Inorganic fertilizer }\end{array}$ \\
\hline T5 & $\begin{array}{l}50 \% \text { Soil : } 50 \% \text { Compost With } 25 \% \\
\text { Inorganic fertilizer }\end{array}$ \\
\hline
\end{tabular}

Compost production. The compost was produced as described by (Baharuddin et al., 2009) with a mixed ratio of 1:1 pressed-shredded empty fruit bunch (EFB) and thickened palm oil mill 
effluent anaerobic sludge, for a period of up to 40 days, to ensure its maturity.

Plant growth. The growth of the seedlings was monitored at three, six and eight months after transplanting by recording the girth size, plant height, frond number, frond length and dry weight. The greenness of the oil palm seedling leaves or the chlorophyll content was measured by using a SPAD 502 Chlorophyll meter. The planting media were sent to the laboratory to be analysed for their nutrient content at the beginning and at the end of the experiment. Leaf samples were harvested from frond number 3 for nutrient analysis at the end of the study by drying the sampled leaves. For biomass determination, the fronds and the girths were separated and dried at $70^{\circ} \mathrm{C}$ in an oven until constant weight.

Soil properties and nutrient content. Soil having weight of $20.00 \mathrm{~g}$ was placed into a plastic bottle. Distilled water was added into the bottle. The soil was shaken intermittently for one hour and left to stand overnight. The $\mathrm{pH}$ was calibrated by using buffers of $\mathrm{pH} 4.00$ and $\mathrm{pH} 7.00$. Basic exchangeable cations were extracted and determined by electrolyte solution in $0.01 \mathrm{M} \mathrm{KCl}$. $\mathrm{CEC}$ was determined with ammonium acetate and the determination of ammonium ions in the soil was done using the colorimetric method. The total organic $\mathrm{C}$ content of the soil was determined by using the Walkley and Black titration method (Gelman et al., 2012). The total $\mathrm{N}$ content of the soil was determined using alkaline phenol and hypochlorite. In order to measure the P content, the soil sample was first digested for $13 / 4$ hours by using a Block Digester at $200^{\circ} \mathrm{C}$ and the analysis of $\mathrm{P}$ was then done using the Auto-Analyser. Determination of soil exchangeable cations (K, $\mathrm{Ca}, \mathrm{Mg}$, and $\mathrm{Na}$ ) was done by using $1 \mathrm{M}$ ammonium acetate. The potassium, magnesium and calcium determinations were done on the AAS by pipetting $2 \mathrm{ml}$ of the original solution and adding $20 \mathrm{ml}$ of $825 \mathrm{ppm}$ Strontium nitrate by using the Auto-Diluter 111. The sodium determination was done using the original solution and reading on the AAS (Sime Darby Research 2018).
Root analysis. The remaining soil adhering to the roots was removed by washing with water. The roots were separated according to their classes, namely the primary and the secondary roots. A digital calliper was used to measure the root diameter. The dry weights of the root samples were recorded after drying them in the oven at 70 $80^{\circ} \mathrm{C}$ until constant weight (Rosenani et al., 2016).

Statistical analysis. Data on the soil properties and nutrient depletion were subjected to analysis of variance (ANOVA) using the SAS Software Windows Version 9.4 (SAS, 2014). Tukey at $P \leq 0.05$ was used to test for significant difference between the treatments. The experiment was arranged in a Randomized Complete Block Design (RCBD) with five treatments and four replications.

\section{RESULTS}

Plant physical characteristics. Figure 1 shows the diagram of oil palm nursery stage comprising of the top part (leaves and rachis) and lower part (girth, primary root and secondary root). Figure 2 shows the plant growth after eight months under the different treatments. Table 2 shows the plant physical characteristic measurements taken after eight months. Table 2 shows plant height, girth size, chlorophyll content and frond length were significantly different on plant physical characteristic. The oil palm seedlings plant height in Treatments 2, 3, 4 and 5 planting media were significantly taller than the control seedlings in the main nursery. The biggest girth size belongs to Treatment 2 and the lowest Treatment 1 . The frond lengths in Treatments 2, 3, 4 and 5 were longer and comparable with the oil palm seedlings at the main nursery in the control Treatment 1. The chlorophyll content was high in Treatment 2, followed by Treatment 3 , Treatment 4 , Treatment 5 and the lowest in Treatment 1 . However, the frond production, and frond number of the leaflet readings taken at the end of the study did not show any significant difference in the values among the treatments tested. 
Table 2. Oil palm main nursery plant characteristics under different treatments.

\begin{tabular}{|c|c|c|c|c|c|c|c|}
\hline & Treatments & $\begin{array}{l}\text { Plant height } \\
\text { (cm) }\end{array}$ & $\begin{array}{c}\text { Frond } \\
\text { production }\end{array}$ & $\begin{array}{l}\text { Girth Size } \\
\quad(\mathrm{cm})\end{array}$ & $\begin{array}{l}\text { Chlorophyll } \\
\text { content } \\
\text { (SPAD unit) }\end{array}$ & $\begin{array}{l}\text { Frond length } \\
\qquad(\mathrm{cm})\end{array}$ & $\begin{array}{c}\text { Frond } \\
\text { number } \\
\text { of leaflet } \\
(\%)\end{array}$ \\
\hline $\mathrm{T} 1$ & $\begin{array}{l}100 \% \text { Soil With } \\
100 \% \text { Inorganic } \\
\text { fertilizer }\end{array}$ & $90.35 \pm 5.89 \mathrm{~b}$ & $17 \pm 0.66 \mathrm{a}$ & $6.31 \pm 0.05 b$ & $52.49 \pm 7.75 b$ & $55.48 \pm 3.52 b$ & $24 \pm 1.25 \mathrm{a}$ \\
\hline $\mathrm{T} 2$ & $\begin{array}{c}50 \% \text { Soil: } 50 \% \\
\text { Compost With } \\
\text { 100\% Inorganic } \\
\text { fertilizer }\end{array}$ & $101.56 \pm 7.92 \mathrm{ab}$ & $18 \pm 0.50 \mathrm{a}$ & $7.62 \pm 0.56 b$ & $59.66 \pm 6.33 a$ & $64.30 \pm 6.23 \mathrm{ab}$ & $26 \pm 2.63 \mathrm{a}$ \\
\hline T3 & $\begin{array}{c}50 \% \text { Soil: } 50 \% \\
\text { Compost With } \\
\text { 75\% Inorganic } \\
\text { fertilizer } \\
\end{array}$ & $101.56 \pm 7.92 \mathrm{ab}$ & $18 \pm 0.50 \mathrm{a}$ & $7.62 \pm 0.56 b$ & $59.66 \pm 6.33 \mathrm{a}$ & $64.30 \pm 6.23 \mathrm{ab}$ & $26 \pm 2.63 a$ \\
\hline $\mathrm{T} 4$ & $\begin{array}{l}50 \% \text { Soil: } 50 \% \\
\text { Compost With } \\
50 \% \text { Inorganic } \\
\text { fertilizer }\end{array}$ & $104.34 \pm 2.04 \mathrm{a}$ & $17 \pm 0.23 \mathrm{a}$ & $7.53 \pm 0.28 b$ & $57.89 \pm 5.73 \mathrm{a}$ & $69.73 \pm 7.21 \mathrm{ab}$ & $26 \pm 1.41 \mathrm{a}$ \\
\hline T5 & $\begin{array}{c}50 \% \text { Soil: } 50 \% \\
\text { Compost With } \\
\text { 25\% Inorganic } \\
\text { fertilizer }\end{array}$ & $104.42 \pm 7.37 \mathrm{a}$ & $17 \pm 0.77 \mathrm{a}$ & $7.50 \pm 0.53 b$ & $59.02 \pm 4.93 \mathrm{a}$ & $63.60 \pm 4.67 \mathrm{ab}$ & $27 \pm 0.58 \mathrm{a}$ \\
\hline
\end{tabular}

Note: Means with the same letter column are not significantly different at $p<0.05$ according to Tukey ( $\mathrm{n}=4)$.

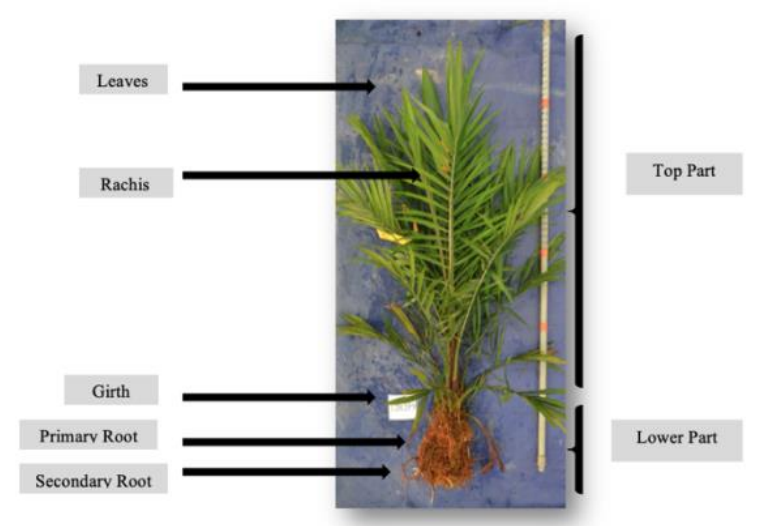

Figure 1. Growth of main nursery seedling at 32th week at nursery stage.

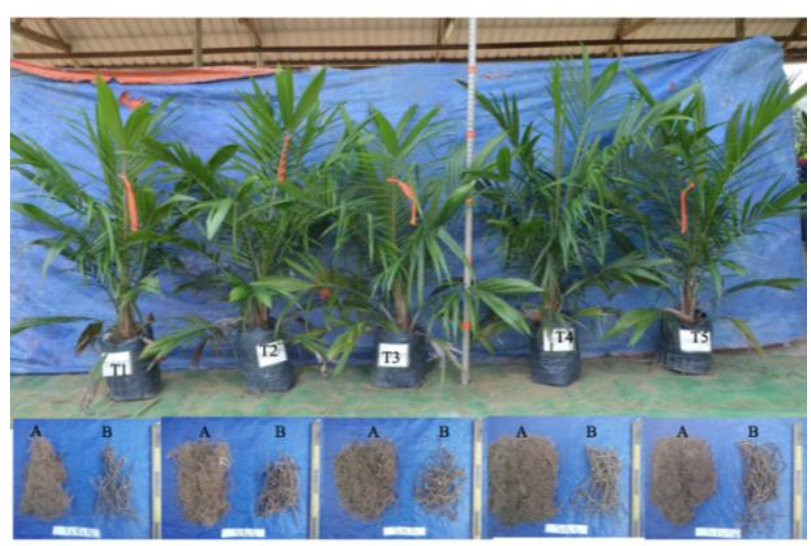

Figure 2. Growth of main nursery seedling at 8 months (32th week) at nursery stage (A) primary $\operatorname{root}(\mathrm{B})$.
Physicochemical characteristic of growth media. Table 3 shows the result on macronutrient analysis on media. The $\mathrm{pH}$ and organic carbon are significantly different between before treatment and after treatment. The $\mathrm{pH}$ value before treatment in $100 \%$ soil was 4.15 and that of $50 \%$ soil: $50 \%$ compost was 4.5 . After treatment, the $\mathrm{pH}$ values in Treatment 1 and Treatment 5 decreased, while treatment with mixed media $50 \%$ soil: $50 \%$ compost in Treatments 2, 3, and 4 showed stable values between 4.01-4.10. Organic carbon before treatment with $50 \%$ soil: $50 \%$ compost had a very high value compared to $100 \%$ soil. The CEC was not significantly different among treatments. Result on macronutrients $\mathrm{N}$, total $\mathrm{P}$, available $\mathrm{P}$, $\mathrm{K}, \mathrm{Ca}, \mathrm{Mg}$ and $\mathrm{Al}$ are all significantly different at $p>0.05$. The concentration of $\mathrm{N}$ in $50 \%$ soil: $50 \%$ compost was high compared to $100 \%$ soil. At the end of the experiment $\mathrm{N}$ content in all Treatments $1,2,3,4$, and 5 ranged from $0.06-0.18 \%$. The same was observed in total $\mathrm{P}$ value in $50 \%$ soil: $50 \%$ compost. Total $\mathrm{P}$ in Treatments 2, 3, 4 and 5 are high compared to Treatment 1. Addition of compost to the media can maintain and increase the amount total of $\mathrm{P}$. The result on available $\mathrm{P}$ has the same pattern with total P. However a different result was observed on $\mathrm{K}$, with little change before and after treatment. Results on 
micronutrient analysis of media, $\mathrm{B}, \mathrm{Zn}, \mathrm{Mn}$, and Si showed significant differences between before and after treatment as shown in Table 4. Interestingly, results on $\mathrm{B}, \mathrm{Fe}, \mathrm{Zn}$, and $\mathrm{Mn}$ showed treatment with $50 \%$ soil: $50 \%$ compost gave the highest values, in contrast with 100\% soil with the lowest values. After treatment, the results for $\mathrm{B}, \mathrm{Fe}, \mathrm{Zn}$ and $\mathrm{Mn}$ showed stable values in media with compost addition.

Table 3. Effects of compost as mixed media on macronutrient chemical characteristics of the main oil palm nursery.

\begin{tabular}{|c|c|c|c|c|c|c|c|c|c|c|c|}
\hline \multirow{2}{*}{\multicolumn{2}{|c|}{ Treatments }} & $\mathrm{pH}$ & Org C & CEC & $\mathbf{N}$ & Total P & Avail P & $\mathbf{K}$ & $\mathrm{Ca}$ & $\mathrm{Mg}$ & Al \\
\hline & & & $(\%)$ & $\begin{array}{c}(\mathrm{cmol}(+) \\
/ \mathrm{kg})\end{array}$ & $(\%)$ & \multicolumn{6}{|c|}{$\begin{array}{c}\mathrm{EXCH} \\
\mathrm{cmol}(+) / \mathrm{kg}\end{array}$} \\
\hline \multicolumn{12}{|c|}{ Before } \\
\hline & $00 \%$ Soil & $\begin{array}{l}4.15 \pm \\
013 b\end{array}$ & $\begin{array}{c}1.31 \pm 0 \\
10 \mathrm{c}\end{array}$ & $\begin{array}{c}4.23 \pm 0 \\
21 \mathrm{a}\end{array}$ & $\begin{array}{c}0.15 \pm 0 \\
15 b\end{array}$ & $\begin{array}{c}44.33 \pm 5 . \\
13 \mathrm{e}\end{array}$ & $\begin{array}{c}10.50 \pm 1 \\
91 \mathrm{c}\end{array}$ & $\begin{array}{r}0.07 \pm \\
0.01 \mathrm{a}\end{array}$ & $\begin{array}{c}1.30 \pm \\
0.12 \mathrm{~b} \\
\mathrm{c}\end{array}$ & $\begin{array}{c}0.17 \pm \\
0.01 \mathrm{e}\end{array}$ & $\begin{array}{c}0.37 \pm 0 \\
03 \mathrm{~b}\end{array}$ \\
\hline & $\begin{array}{l}\text { o Soil : } 50 \% \\
\text { Compost }\end{array}$ & $\begin{array}{l}4.5 \pm 0 \\
.08 \mathrm{a}\end{array}$ & $\begin{array}{c}7.59 \pm 0 . \\
12 \mathrm{a}\end{array}$ & $\begin{array}{c}4.12 \pm 0 . \\
12 \mathrm{a}\end{array}$ & $\begin{array}{c}0.38 \pm 0 . \\
01 \mathrm{a}\end{array}$ & $\begin{array}{c}973.33 \pm \\
19.76 \mathrm{a}\end{array}$ & $\begin{array}{c}27.50 \pm 2 . \\
08 c\end{array}$ & $\begin{array}{l}0.03 \pm \\
0.01 \mathrm{~b}\end{array}$ & $\begin{array}{r}2.13 \pm \\
0.02 \mathrm{a}\end{array}$ & $\begin{array}{r}2.11 \pm \\
0.03 \mathrm{a}\end{array}$ & $\begin{array}{c}0.65 \pm 0 . \\
01 \mathrm{a}\end{array}$ \\
\hline \multicolumn{12}{|c|}{ After } \\
\hline T1 & $\begin{array}{c}100 \% \text { Soil } \\
\text { With } 100 \% \\
\text { Inorganic } \\
\text { fertilizer }\end{array}$ & $\begin{array}{l}3.77 \pm \\
0.13 c\end{array}$ & $\begin{array}{c}1.20 \pm 0 \\
13 \mathrm{c}\end{array}$ & $\begin{array}{c}5.23 \pm 0 . \\
33 \mathrm{a}\end{array}$ & $\begin{array}{c}0.06 \pm 0 \\
01 \mathrm{a}\end{array}$ & $\begin{array}{c}200.33 \pm \\
12.0 \mathrm{~d}\end{array}$ & $\begin{array}{c}64.00 \pm 17 \\
.32 \mathrm{~b}\end{array}$ & $\begin{array}{r}0.08 \pm \\
0.01 \mathrm{a}\end{array}$ & $\begin{array}{l}1.66 \pm \\
0.26 \mathrm{~b}\end{array}$ & $\begin{array}{c}0.44 \pm \\
0.09 \mathrm{~d} \\
\mathrm{e}\end{array}$ & $\begin{array}{c}0.20 \pm 0 . \\
02 \mathrm{c}\end{array}$ \\
\hline $\mathrm{T} 2$ & $\begin{array}{c}50 \% \text { Soil : } \\
50 \% \\
\text { Compost } \\
\text { With } 100 \% \\
\text { Inorganic } \\
\text { fertilizer }\end{array}$ & $\begin{array}{l}4.10 \pm \\
0.09 \mathrm{~b}\end{array}$ & $\begin{array}{c}4.23 \pm 1 \\
12 \mathrm{~b}\end{array}$ & $\begin{array}{c}5.10 \pm 2 . \\
36 \mathrm{a}\end{array}$ & $\begin{array}{c}0.18 \pm 0 \\
05 \mathrm{a}\end{array}$ & $\begin{array}{c}466.67 \pm \\
17.67 \mathrm{bc}\end{array}$ & $\begin{array}{c}147.00 \pm 7 \\
.73 a\end{array}$ & $\begin{array}{c}0.06 \pm \\
0.03 \mathrm{a} \\
\mathrm{b}\end{array}$ & $\begin{array}{l}1.27 \pm \\
0.17 \mathrm{c}\end{array}$ & $\begin{array}{l}1.37 \pm \\
0.02 \mathrm{~b}\end{array}$ & $\begin{array}{c}0.35 \pm 0 \\
02 \mathrm{~b}\end{array}$ \\
\hline T3 & $\begin{array}{c}50 \% \text { Soil : } \\
50 \% \\
\text { Compost } \\
\text { With } 75 \% \\
\text { Inorganic } \\
\text { fertilizer }\end{array}$ & $\begin{array}{l}4.06 \pm \\
0.06 \mathrm{~b}\end{array}$ & $\begin{array}{c}5.12 \pm 0 . \\
85 b\end{array}$ & $\begin{array}{c}4.04 \pm 0 . \\
24 \mathrm{a}\end{array}$ & $\begin{array}{c}0.18 \pm 0 \\
03 \mathrm{a}\end{array}$ & $\begin{array}{c}544.67 \pm \\
34.39 \mathrm{~b}\end{array}$ & $\begin{array}{c}151.75 \pm 2 \\
.06 a\end{array}$ & $\begin{array}{c}0.05 \pm \\
0.01 \mathrm{a} \\
\mathrm{b}\end{array}$ & $\begin{array}{l}0.76 \pm \\
0.16 \mathrm{~d}\end{array}$ & $\begin{array}{c}1.00 \pm \\
0.36 b \\
c\end{array}$ & $\begin{array}{c}0.31 \pm 0 \\
06 \mathrm{bc}\end{array}$ \\
\hline $\mathrm{T} 4$ & $\begin{array}{c}50 \% \text { Soil : } \\
50 \% \\
\text { Compost } \\
\text { With } 50 \% \\
\text { Inorganic } \\
\text { fertilizer }\end{array}$ & $\begin{array}{l}4.01 \pm \\
0.08 \mathrm{~b}\end{array}$ & $\begin{array}{c}4.16 \pm 1 \\
28 \mathrm{~b}\end{array}$ & $\begin{array}{c}4.62 \pm 1 \\
38 \mathrm{a}\end{array}$ & $\begin{array}{c}0.15 \pm 0 \\
03 \mathrm{a}\end{array}$ & $\begin{array}{c}544.67 \pm \\
34.12 \mathrm{~b}\end{array}$ & $\begin{array}{c}166.75 \pm 1 \\
8.84 \mathrm{a}\end{array}$ & $\begin{array}{c}0.05 \pm \\
0.02 \mathrm{a} \\
\mathrm{b}\end{array}$ & $\begin{array}{l}0.42 \pm \\
0.08 \mathrm{~d}\end{array}$ & $\begin{array}{c}0.74 \pm \\
0.26 \mathrm{c} \\
\mathrm{d}\end{array}$ & $\begin{array}{c}0.27 \pm 0 \\
11 \mathrm{bc}\end{array}$ \\
\hline T5 & $\begin{array}{c}50 \% \text { Soil : } \\
50 \% \\
\text { Compost } \\
\text { With } 25 \% \\
\text { Inorganic } \\
\text { fertilizer }\end{array}$ & $\begin{array}{c}3.98 \pm \\
0.02 b \\
c\end{array}$ & $\begin{array}{c}3.78 \pm 0 \\
71 \mathrm{~b}\end{array}$ & $\begin{array}{c}4.68 \pm 1 \\
43 a\end{array}$ & $\begin{array}{c}0.14 \pm 0 \\
02 \mathrm{a}\end{array}$ & $\begin{array}{c}396.33 \pm \\
9.01 \mathrm{c}\end{array}$ & $\begin{array}{c}59.75 \pm 21 \\
.75 b\end{array}$ & $\begin{array}{c}0.06 \pm \\
0.01 \mathrm{a} \\
\mathrm{b}\end{array}$ & $\begin{array}{l}0.66 \pm \\
0.10 \mathrm{~d}\end{array}$ & $\begin{array}{c}0.62 \pm \\
0.13 c \\
d\end{array}$ & $\begin{array}{c}0.22 \pm 0 \\
04 \mathrm{c}\end{array}$ \\
\hline
\end{tabular}

Note: Means with the same letter column are not significantly different at $p<0.05$ according to Tukey ( $\mathrm{n}=4)$. 
Table 4. Effect of compost as mixed media on macronutrient chemical characteristics by oil palm main nursery.

\begin{tabular}{|c|c|c|c|c|c|c|}
\hline \multirow{2}{*}{\multicolumn{2}{|c|}{ Treatments }} & $\mathbf{B}$ & $\mathrm{Fe}$ & $\mathrm{Zn}$ & Mn & Si \\
\hline & & \multicolumn{5}{|c|}{$\mathrm{mg} / \mathrm{kg}$} \\
\hline \multicolumn{7}{|l|}{ Before } \\
\hline & $100 \%$ Soil & $0.59 \pm 0.05 \mathrm{c}$ & $338.15 c$ & $2.37 \pm 0.34 b$ & $1.22 \pm 0.07 \mathrm{c}$ & $125.55 \pm 10.31 \mathrm{a}$ \\
\hline \multicolumn{2}{|c|}{$50 \%$ Soil : $50 \%$ Compost } & $3.25 \pm 0.04 \mathrm{a}$ & $455.46 \mathrm{bc}$ & $27.85 \pm 7.05 \mathrm{a}$ & $28.04 \pm 0.90 \mathrm{c}$ & $0.23 \pm 0.02 \mathrm{~b}$ \\
\hline \multicolumn{2}{|l|}{ After } & & & & & \\
\hline T1 & $\begin{array}{l}100 \% \text { Soil With } \\
100 \% \text { Inorganic } \\
\text { fertilizer }\end{array}$ & $0.52 \pm 0.10 \mathrm{c}$ & $581.35 \mathrm{ab}$ & $1.68 \pm 0.06 \mathrm{~b}$ & $157.92 \pm 6.82 \mathrm{a}$ & $3.91 \pm 0.22 \mathrm{~b}$ \\
\hline $\mathrm{T} 2$ & $\begin{array}{c}50 \% \text { Soil : } 50 \% \\
\text { Compost With } 100 \% \\
\text { Inorganic fertilizer }\end{array}$ & $1.55 \pm 0.32 b$ & $517.95 \mathrm{ab}$ & $1.98 \pm 0.07 b$ & $110.49 \pm 18.97 b$ & $5.68 \pm 0.76 b$ \\
\hline T3 & $\begin{array}{c}50 \% \text { Soil : } 50 \% \\
\text { Compost With } 75 \% \\
\text { Inorganic fertilizer }\end{array}$ & $1.62 \pm 0.23 \mathrm{~b}$ & $577.95 \mathrm{ab}$ & $2.00 \pm 0.06 \mathrm{~b}$ & $112.43 \pm 10.43 b$ & $4.42 \pm 0.76 \mathrm{~b}$ \\
\hline T4 & $\begin{array}{l}50 \% \text { Soil : } 50 \% \\
\text { Compost With } 50 \% \\
\text { Inorganic fertilizer }\end{array}$ & $1.35 \pm 0.22 b$ & $589.56 \mathrm{ab}$ & $1.91 \pm 0.05 b$ & $127.56 \pm 23.63 \mathrm{ab}$ & $4.07 \pm 1.47 \mathrm{~b}$ \\
\hline T5 & $\begin{array}{l}50 \% \text { Soil : } 50 \% \\
\text { Compost With } 25 \% \\
\text { Inorganic fertilizer }\end{array}$ & $1.5 \pm 0.15 b$ & $674.77 \mathrm{a}$ & $1.93 \pm 0.04 b$ & $128.95 \pm 16.28 \mathrm{ab}$ & $4.44 \pm 0.61 b$ \\
\hline
\end{tabular}

Note: Means with the same letter column are not significantly different at $p<0.05$ according to Tukey ( $\mathrm{n}=4)$.

Dry weights of leaf, rachis, girth and root. The results presented in Table 5 showed that the dry weight of rachis was not significantly different among the treatments. The highest dry weight of leaves within all media mixed and the lowest on control know as Treatment 1, 100\% soil with $100 \%$ inorganic fertilizer. Result on dry weight of girth size, total of shoot and total of root same trend with dry weight of leaves, with media mixed got high dry weight. The total root showed that Treatment 5 had the best root followed by Treatment 4, Treatment 3 and Treatment 2, with the lowest in Treatment 1 (control). Figure 3 shows that the primary and secondary roots were significantly different in the main nursery of the oil palm. The primary and second roots of seedlings treated with $50 \%$ compost showed better results compared to the control. (a)

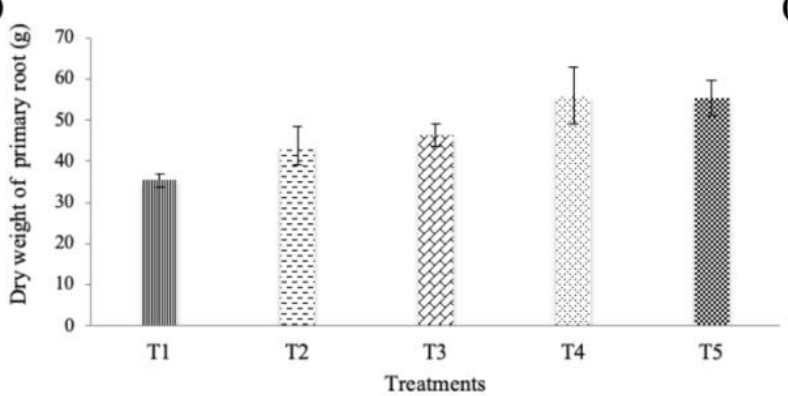

(b)

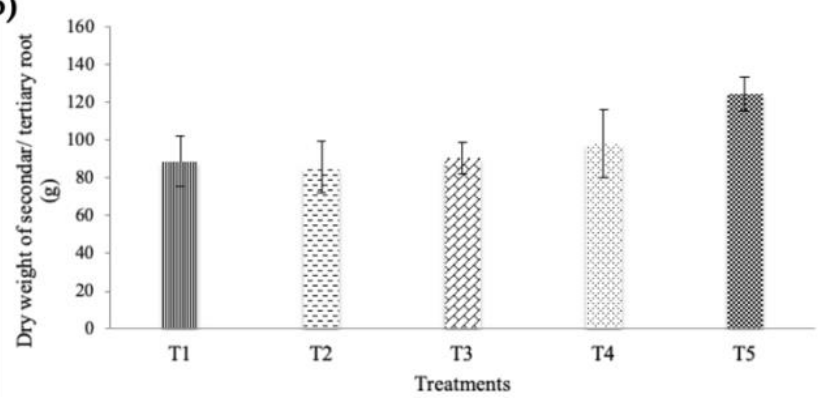

T1 $\quad 100 \%$ Soil With $100 \%$ Inorganic fertilizer

T2 $50 \%$ Soil : $50 \%$ Compost With $100 \%$ Inorganic fertilizer

T3 $50 \%$ Soil : 50\% Compost With $75 \%$ Inorganic fertilizer

T4 $50 \%$ Soil : $50 \%$ Compost With 50\% Inorganic fertilizer

T5 $50 \%$ Soil : 50\% Compost With 25\% Inorganic fertilizer

Figure 3. Effects of compost application on (a) primary roots and (b) secondary/tertiary roots of seedlings in the main oil palm nursery. Bars with different lowercase letter differ significantly $(p<0.05)$. 
Table 5. Effects of compost as mix media on dry weights of seedlings in the oil palm main nursery

\begin{tabular}{|c|c|c|c|c|c|c|c|}
\hline & Treatments & $\begin{array}{l}\text { Dry Weight } \\
\text { of Leaves } \\
\text { (g/plant) }\end{array}$ & $\begin{array}{c}\text { Dry Weight } \\
\text { of Rachis } \\
\text { (g/plant) }\end{array}$ & $\begin{array}{c}\text { Dry Weight of } \\
\text { Girth } \\
\text { (g/plant) }\end{array}$ & $\begin{array}{l}\text { Total of } \\
\text { Shoot } \\
\text { (g/plant) }\end{array}$ & $\begin{array}{c}\text { Total of } \\
\text { Root } \\
\text { (g/plant) }\end{array}$ & $\begin{array}{l}\text { Root: } \\
\text { Shoot }\end{array}$ \\
\hline T1 & $\begin{array}{c}100 \% \text { Soil With } \\
100 \% \text { Inorganic } \\
\text { fertilizer }\end{array}$ & $\begin{array}{c}177.27 \pm 4.50 \\
\mathrm{~b}\end{array}$ & $\begin{array}{c}133.43 \pm 22.6 \\
4 a\end{array}$ & $102.98 \pm 12.71 b$ & $\begin{array}{c}310.70 \pm 24.1 \\
6 \mathrm{c}\end{array}$ & $\begin{array}{c}128.93 \pm 1.59 \\
b\end{array}$ & $\begin{array}{c}0.41 \pm 0.03 \mathrm{a} \\
\mathrm{b}\end{array}$ \\
\hline $\mathrm{T} 2$ & $\begin{array}{c}50 \% \text { Soil: } 50 \% \\
\text { Compost With } \\
\text { 100\% Inorganic } \\
\text { fertilizer }\end{array}$ & $\begin{array}{c}101.56 \pm 7.92 \mathrm{a} \\
\mathrm{b}\end{array}$ & $18 \pm 0.50 \mathrm{a}$ & $7.62 \pm 0.56 b$ & $59.66 \pm 6.33 \mathrm{a}$ & $\begin{array}{c}64.30 \pm 6.23 \mathrm{a} \\
\mathrm{b}\end{array}$ & $26 \pm 2.63 a$ \\
\hline T3 & $\begin{array}{c}50 \% \text { Soil : } 50 \% \\
\text { Compost With } \\
100 \% \text { Inorganic } \\
\text { fertilizer }\end{array}$ & $\begin{array}{c}215.00 \pm 21.3 \\
2 \mathrm{a}\end{array}$ & $\begin{array}{c}163.34 \pm 12.7 \\
1 \mathrm{a}\end{array}$ & $\begin{array}{c}118.58 \pm 25.00 \mathrm{a} \\
\mathrm{b}\end{array}$ & $378.37 \pm 8.66 \mathrm{a}$ & $\begin{array}{c}136.56 \pm 4.86 \\
b\end{array}$ & $\begin{array}{c}0.36 \pm 0.02 \\
\mathrm{~b}\end{array}$ \\
\hline T4 & $\begin{array}{c}50 \% \text { Soil: } 50 \% \\
\text { Compost With } \\
50 \% \text { Inorganic } \\
\text { fertilizer } \\
\end{array}$ & $104.34 \pm 2.04 \mathrm{a}$ & $17 \pm 0.23 a$ & $7.53 \pm 0.28 b$ & $57.89 \pm 5.73 \mathrm{a}$ & $\begin{array}{c}69.73 \pm 7.21 \mathrm{a} \\
\mathrm{b}\end{array}$ & $26 \pm 1.41 \mathrm{a}$ \\
\hline T5 & $\begin{array}{c}50 \% \text { Soil : } 50 \% \\
\text { Compost With } \\
75 \% \text { Inorganic } \\
\text { fertilizer }\end{array}$ & $\begin{array}{c}187.13 \pm 3.91 \mathrm{a} \\
\mathrm{b}\end{array}$ & $\begin{array}{c}132.70 \pm 16.6 \\
84 \mathrm{a}\end{array}$ & $117.46 \pm 8.60 \mathrm{ab}$ & $\begin{array}{c}319.83 \pm 16.0 \\
0 \mathrm{bc}\end{array}$ & $\begin{array}{c}134.57 \pm 5.95 \\
b\end{array}$ & $\begin{array}{c}0.42 \pm 0.04 a \\
b\end{array}$ \\
\hline
\end{tabular}

Note: Means with the same letter column are not significantly different at $p<0.05$ according to Tukey ( $\mathrm{n}=4)$.

Foliar nutrients. The results presented in Table 6 showed that the foliar nutrients from the main nursery were only significantly different for $\mathrm{N}$ and $\mathrm{P}$. The $\mathrm{K}, \mathrm{Ca}, \mathrm{Mg}, \mathrm{S}, \mathrm{B}, \mathrm{Cu}, \mathrm{Fe}$, and $\mathrm{Zn}$ contents did not show any significant difference among all the planting media tested. It was postulated that the higher total $\mathrm{N}$ uptake in the leaf could be due to the supplementary nutrient content of the compost itself and the optimum $\mathrm{C}$ : $\mathrm{N}$ ratio (Robbins, 2013). The value for $\mathrm{N}$ was the highest in Treatment 2, followed by Treatments 3 and 4 .

Cost of material. Table 7 shows the material cost for oil palm planting media at nursery stage. The highest cost was for $100 \%$ soil with $100 \%$ inorganic fertilizer (Treatment1) as control at RM6.04 per polybag, followed by 50\% soil: 50 compost with $100 \%$ inorganic fertilizer at RM5.20 (Treatment 2). Treatment 3 with $50 \%$ soil: $50 \%$ compost with $75 \%$ inorganic fertilizer was RM4.64 per polybag while in Treatment 4 it was RM4.23. The lowest cost was in Treatment 5 at RM3.92 per polybag.

\section{DISCUSSION}

A suitable planting media will have a positive effect on the physical characteristics and vegetative growth of oil palm seedling (Akb et al., 2018). The dry weight of leaves, rachis and shoot to root ratio did not significantly differ among the treatments but the plant height, dry weight of girth and total of root significantly differed, indicating that the addition of composted agricultural waste significantly improved root growth. A similar phenomenon was reported by Al-Busaidi (2013). Nitrogen content has a strong correlation on leaf chlorophyll content, seedling height, root diameter and shoot morphology (Schlemmer et al., 2013; Cuesta et al., 2010; Andivia et al., 2011; Oliet et al., 2009). N availability affected root elongation, root biomass, production and mortality (Kern et al., 2004; Woolfolk and Friend, 2003). Primary root length is reduced by lowering nitrate application (Mounier et al., 2014). Phosphorus (P) is essential for plant growth for root development, new shoot growth, flower development and for providing energy for the plant. P has mixed effect on root collar and plant height (Huda et al., 2007). Starvation of phosphate stimulates lateral root growth, inhibit primary root and enhances root hair production (Shahzad and Amtmann, 2017). Efficient root management can help reduce the requirement of inorganic fertilizers (Shen et al., 2013). From our results, even with the reduction in application to $50 \%$ compost with $50 \%$ soil : $50 \%$ inorganic fertilizer, there was 30\% increase 
in the total root biomass compared to $100 \%$ soil with 100\% inorganic fertilizer. The diameters of the primary and secondary roots were significantly increased by using the mixed medium of $\mathrm{T} 4$ as $50 \%$ soil: $50 \%$ compost with $50 \%$ inorganic fertilizer as shown in Figure 1. This is supported by Zuraidah et al. (2015) who reported that the secondary root can penetrate the soil deeper to extract the nutrients on oil palm nursery. Root development on compost has better penetration because the soil mixed with compost had larger pores and less compaction compared to $100 \%$ soil as media (Celik et al., 2010). This indicated that the root structure with compost could penetrate the soil deeper and absorb more nutrients as compared to the roots without compost. Lord and Betitis (2007) reported that oil palm seedlings raised plant height on compost with $75 \%, 50 \%$ and $25 \%$ of normal fertilizer regime had both widths and heights which were significantly greater than the soil at $100 \%$ of normal regime. Compost addition improved soil aeration and pore space. Compost application increased soil C content and also improved soil aggregation contributing to better crop growth (Rivenshield and Bassuk, 2007). These further highlighted the importance of compost in the growth medium to alleviate soil C content and to improve soil physical properties (Norizan et al., 2016). Adding compost also improved the chemical properties of the growth medium as shown by various studies, which reported the ability of the compost to reduce soil acidity (Valarini et al., 2009 and Giannakis et al., 2014). Availability of nutrients depend on $\mathrm{pH}$ (Michael et al., 2012). The C content of the medium increased significantly after treatment, treatment with $50 \%$ soil: $50 \%$ compost being $7.59 \%$ as compared to $100 \%$ soil at $1.31 \%$. Abad et al. (2001) reported a similar pattern when compost was added to ornamental potted plant. Organic carbon for treatment in $100 \%$ soil with $100 \%$ inorganic fertiliser was $1.20 \%$, compared to treatment in $50 \%$ soil : $50 \%$ compost which had a higher organic content from $3.78 \%-5.12 \%$, probably due to additional binding sites for exchangeable bases and decreased the availability of polycations like $\mathrm{Fe}^{2+}$ and $\mathrm{Al}^{3+}(\mathrm{Xu}$ et al., 2006). Thus, the addition of compost which is rich in negative functionalities could stabilize the $\mathrm{pH}$ value of the growth medium (Goh et al., 1997). Besides, the high contents of alkaline elements (excess cations), particularly $\mathrm{Ca}$ and $\mathrm{Mg}$ which are present in the compost, could react with the free $\mathrm{H}^{+}$. This explained the liming effects of compost and the decarboxylation of organic anions during decomposition, improving the $\mathrm{pH}$ of the growth medium. Soil chemical characteristic is an important consideration in assessing the suitability of a soil for oil palm cultivation and the requirement for mineral fertilizers. Aluminum toxicity is recognized as a common concern in mineral soils at a low $\mathrm{pH}$, but this is usually not a problem in organic-based mixed media (Xu et al., 2006). This might be due to the depletion of the top soil in the old running nursery. At the end of experiment, both compost-based treatments by $50 \%$ soil: $50 \%$ compost with $75 \%$ inorganic fertilizer and by $50 \%$ soil: $50 \%$ compost with $50 \%$ inorganic fertilizer showed the highest values in total $\mathrm{N}$ compared to the other treatments, a reflection of the high $\mathrm{N}$ uptake by the leaves. Furthermore, compost could also supply the seedlings with additional $\mathrm{N}, \mathrm{P}, \mathrm{Ca}, \mathrm{Mg}$, and $\mathrm{Zn}$, functioning as a source of nutrients and trace elements besides the inorganic fertilizers. Another mechanism could be that compost retained the nutrients in the growth medium by surface adsorption, thus increasing the nutrient recovery (Jouquet et al., 2011). The cost of material was similar as reported by Mohammad (2014).The increasing production cost of seedlings is one of the main issues to a nursery operator. With the calculated cost of material and good performance of seedlings produced, the findings and results of this study can be used as a basis for commercial venture. 
Table 6. Effects of compost as mix media on foliar nutrient in the main oil palm nursery.

\begin{tabular}{|c|c|c|c|c|c|c|c|c|c|c|c|c|}
\hline \multirow{2}{*}{\multicolumn{2}{|c|}{ Treatments }} & Total N & $\mathbf{P}$ & $\mathbf{K}$ & $\mathrm{Ca}$ & $\mathrm{Mg}$ & $\mathrm{Cl}$ & $\mathrm{S}$ & B & $\mathrm{Cu}$ & $\mathrm{Fe}$ & $\mathrm{Zn}$ \\
\hline & & $(\%)$ & & & $\begin{array}{c}\text { EXCH } \\
\mathrm{cmol}(+) / 1\end{array}$ & & & \multicolumn{5}{|c|}{$\mathrm{mg} / \mathrm{kg}$} \\
\hline $\mathrm{T} 1$ & $\begin{array}{c}100 \% \text { Soil With } \\
\text { 100\% Inorganic } \\
\text { fertilizer }\end{array}$ & $\begin{array}{c}2.71 \pm 0.1 \\
2 \mathrm{ab}\end{array}$ & $\frac{0.17 \pm 0.01 \mathrm{a}}{\mathrm{b}}$ & $\begin{array}{c}0.90 \pm 0.0 \\
3 \mathrm{a}\end{array}$ & $0.63 \pm 0.07 \mathrm{a}$ & $\begin{array}{c}0.26 \pm 0.0 \\
3 \mathrm{a}\end{array}$ & $0.88 \pm 0.04 \mathrm{a}$ & $\begin{array}{c}0.13 \pm 0.0 \\
1 \mathrm{~b}\end{array}$ & $\begin{array}{c}16.00 \pm 0.82 \\
\mathrm{a}\end{array}$ & $\begin{array}{c}1.55 \pm 0.3 \\
6 \mathrm{a}\end{array}$ & $\begin{array}{c}96.11 \pm 3.15 \\
\mathrm{a}\end{array}$ & $\begin{array}{c}19.32 \pm 0 \\
84 \mathrm{a}\end{array}$ \\
\hline $\mathrm{T} 2$ & $\begin{array}{c}50 \% \text { Soil : } 50 \% \\
\text { Compost With } \\
\text { 100\% Inorganic } \\
\text { fertilizer }\end{array}$ & $\begin{array}{c}2.88 \pm 0.0 \\
6 \mathrm{a}\end{array}$ & $0.18 \pm 0.01 \mathrm{a}$ & $\begin{array}{c}1.00 \pm 0.0 \\
8 \mathrm{a}\end{array}$ & $0.65 \pm 0.09 \mathrm{a}$ & $\begin{array}{c}0.28 \pm 0.0 \\
2 \mathrm{a}\end{array}$ & $0.88 \pm 0.03 a$ & $\begin{array}{c}0.14 \pm 0.0 \\
1 \mathrm{a}\end{array}$ & $\begin{array}{c}15.75 \pm 1.26 \\
\mathrm{a}\end{array}$ & $\begin{array}{c}1.74 \pm 0.5 \\
5 \mathrm{a}\end{array}$ & $\begin{array}{c}97.81 \pm 7.80 \\
\mathrm{a}\end{array}$ & $\begin{array}{c}20.11 \pm 1 \\
18 a\end{array}$ \\
\hline T3 & $\begin{array}{c}50 \% \text { Soil : } 50 \% \\
\text { Compost With } \\
75 \% \text { Inorganic } \\
\text { fertilizer }\end{array}$ & $\begin{array}{c}2.84 \pm 0.1 \\
2 \mathrm{ab}\end{array}$ & $\frac{0.17 \pm 0.01 \mathrm{a}}{\mathrm{b}}$ & $\begin{array}{c}0.91 \pm 0.0 \\
2 \mathrm{a}\end{array}$ & $0.73 \pm 0.05 a$ & $\begin{array}{c}0.25 \pm 0.0 \\
1 \mathrm{a}\end{array}$ & $0.88 \pm 0.02 \mathrm{a}$ & $\begin{array}{c}0.14 \pm 0.0 \\
1 \mathrm{a}\end{array}$ & $\begin{array}{c}16.00 \pm 0.82 \\
\mathrm{a}\end{array}$ & $\begin{array}{c}2.38 \pm 0.2 \\
7 \mathrm{a}\end{array}$ & $\begin{array}{c}101.93 \pm 3.7 \\
6 \mathrm{a}\end{array}$ & $\begin{array}{c}21.03 \pm 1 \\
05 \mathrm{a}\end{array}$ \\
\hline $\mathrm{T} 4$ & $\begin{array}{c}50 \% \text { Soil : } 50 \% \\
\text { Compost With } \\
50 \% \text { Inorganic } \\
\text { fertilizer }\end{array}$ & $\begin{array}{c}2.84 \pm 0.0 \\
3 \mathrm{ab}\end{array}$ & $\frac{0.17 \pm 0.01 \mathrm{a}}{\mathrm{b}}$ & $\begin{array}{c}0.91 \pm 0.0 \\
6 \mathrm{a}\end{array}$ & $0.72 \pm 0.04 \mathrm{a}$ & $\begin{array}{c}0.26 \pm 0.0 \\
1 \mathrm{a}\end{array}$ & $0.88 \pm 0.01 \mathrm{a}$ & $\begin{array}{c}0.15 \pm 0.0 \\
1 \mathrm{a}\end{array}$ & $\begin{array}{c}15.50 \pm 1.00 \\
\mathrm{a}\end{array}$ & $\begin{array}{c}2.34 \pm 0.3 \\
2 \mathrm{a}\end{array}$ & $\begin{array}{c}101.55 \pm 3.4 \\
4 \mathrm{a}\end{array}$ & $\begin{array}{c}21.26 \pm 0 \\
38 \mathrm{a}\end{array}$ \\
\hline T5 & $\begin{array}{c}50 \% \text { Soil : } 50 \% \\
\text { Compost With } \\
\text { 25\% Inorganic } \\
\text { fertilizer }\end{array}$ & $\begin{array}{c}2.67 \pm 0.0 \\
3 \mathrm{~b}\end{array}$ & $0.16 \pm 0.01 \mathrm{~b}$ & $\begin{array}{c}0.91 \pm 0.0 \\
4 \mathrm{a}\end{array}$ & $0.66 \pm 0.11 \mathrm{a}$ & $\begin{array}{c}0.24 \pm 0.0 \\
1 \mathrm{a}\end{array}$ & $0.90 \pm 0.01 \mathrm{a}$ & $\begin{array}{c}0.14 \pm 0.0 \\
1 \mathrm{a}\end{array}$ & $14.5 \pm 1.00 \mathrm{a}$ & $\begin{array}{c}2.22 \pm 0.4 \\
1 \mathrm{a}\end{array}$ & $\begin{array}{c}98.91 \pm 2.60 \\
\mathrm{a}\end{array}$ & $\begin{array}{c}19.21 \pm 1 \\
41 \mathrm{a}\end{array}$ \\
\hline
\end{tabular}

Note: Means with the same letter column are not significantly different at $p<0.05$ according to Tukey. 
Table 7. Material cost per polybag of planting media.

\begin{tabular}{clc}
\hline Treatment & \multicolumn{1}{c}{ Percentage soil with inorganic fertilizer } & RM / Polybag \\
\hline T1 & 100\% Soil With 100\% Inorganic fertilizer & RM6.04 \\
T2 & $50 \%$ Soil : 50\% Compost With 100\% Inorganic fertilizer & RM5.20 \\
T3 & $50 \%$ Soil : 50\% Compost With 75\% Inorganic fertilizer & RM4.64 \\
T4 & $50 \%$ Soil : 50\% Compost With 50\% Inorganic fertilizer & RM4.23 \\
T5 & $50 \%$ Soil : 50\% Compost With 25\% Inorganic fertilizer & RM3.92 \\
\hline
\end{tabular}

Topsoil $=$ RM160/ton, Compost $=$ RM150/ton, NPK Blue $=$ RM1300/ton and NPK Yellow $=$ RM1200/ton.

\section{CONCLUSION}

The loss of nutrients from the oil palm nursery system, especially in the main nursery can be high if high inorganic fertilizer rates are applied and the best medium is used. Improvement in nutrient retention should be possible with adaptation of irrigation methods that supply water more efficiently and media as fertilizer and slow release nutrient sources. Replacement of inorganic fertilizer by $50 \%$ compost in the growth medium mixture in the main nursery was able to reduce the application of inorganic fertilizer by as much as $50 \%$, resulting in overall cost reduction. The important finding in this study was that reduction of $50 \%$ inorganic fertilizer using $50 \%$ soil: $50 \%$ compost medium did not affect plant growth. Secondary and tertiary roots, which are important for the absorption of nutrients from the soil, were found to increase in all treatments with $50 \%$ soil: $50 \%$ compost, thus allowing for the reduction of inorganic fertilizer use. Further studies on the use of compost in the mixed growth medium should be done to ascertain the effects of the reduction of inorganic fertilizer on the soil microbial profile.

\section{ACKNOWLEDGEMENTS}

The authors would like to acknowledge JICA-JST Japan and The Ministry of Education Malaysia for the SATREPS (Oil Palm) Research Grant from 2014-2018 (Project Code 6300156). We are grateful to the Senior Vice President R\&D and CEO of Felda Agricultural Services Sdn. Bhd. for their approval of this joint venture project. The authors have declared that no conflict of interest exists.

\section{REFERENCES}

Abad, M., Noguera, P., \& Bures, S. 2001. National inventory of organic wastes for use as growing media for ornamental potted plant production. Bioresource Technology 77: 197-200.

Akb, O., Gk, O., Jc, N., \& Amoah, P. 2018. Growth, dry matter yield and nutrient uptake of oil palm seedlings (Elaies guineensis Jacq.) as affected by different soil ammendments. Advances in Crop Science and Technology 6: 4-7.

Al-Busaidi, K. T. S. 2013. Effects of organic and inorganic fertilizers addition on growth and yield of banana (Musa AAA cv . Malindi ) on a saline and non-saline soil in Oman. Journal of Hotriculture and Forestry 5: 146-155.

Albregts, E. E., \& Chandler, C. K. 1993. Slow release fertilizer rates for strawberry fruit production. Proceedings of the Florida State Horticultural Society 106: 187-189.

Andivia, E., Fernandez, M., Vazquez-Pique, J. 2011. Autumn fertilization of Quercus ilex ssp. ballota (Desf.) Samp. nursery seedlings: Effects on morpho-physiology and field performance. Annals of Forest Science 68:543-553

Ashraf, A. N., Zulkefly, S., Adekunle, S. M., \& Samad, M. Y. A. 2017. Growth and biomass yield of oil palm (Elaeis guineensis) seedlings as influenced by different rates of vermicompost. European Journal of Engineering Research and Science 2: 17-21.

Baharuddin, A. S., Wakisaka, M., Shirai, Y., Abd-Aziz, S., Abdul Rahman N. A., \& Hassan, M. A. 2009. Co-composting of empty fruit bunches and partially treated palm oil mill effluents in pilot scale. International Journal Agricultural Research 4: 69-78.

Celik, I., Gunal, H., Budak, M., \& Akpinar, C. 2010. Geoderma effects of long-term organic and mineral fertilizers on bulk density and penetration resistance in semi-arid Mediterranean soil conditions. Geoderma 160: 236-243.

Cuesta, B., Villar, S. P., Puertolas, J., \& Jacobs, D. F. 2010. Why do large, nitrogen rich seedlings better resist stressful transplanting conditions? A physiological analysis in two functionally contrasting Mediterranean forest species. Forest Ecology Management 260: 71-78.

Gelman, F., Binstock, R., \& Halicz, L. 2012. Application of the Walkley-Black titration for the organic carbon quantification in organic rich sedimentary rocks. Fuel 96: 608-610.

Goh, K. J. 1997. Fertilizer recommendation systems for oil palm: estimating the fertilizer rates. Proceedings of MOSTA best practices workshops: agronomy and crop management, Malaysia, Malaysia, March to August 1-37.

Huda, S. M. S., Sujauddin, M., Shafinat, S., \& Uddin, M. S. 2007. Effects of phosphorus and potassium addition on growth and nodulation of Dalbergia sissoo in the nursery. Journal of Forest Research 18: 279-282.

Jouquet, E., Bloquel, E., Doan, T. T., Ricoy, M., Orange, D., Rumpel, C., \& Duc, T. T. 2011. Do compost and vermicompost improve macronutrient retention and plant 
growth in degraded tropical soils? Compost Science Utilization 19: 15-24.

Kern, C. C., Friend, A. L., Johnson, J. M. F., \& Coleman, M. D. 2004. Fine root dynamics in a developing Populus deltoides plantation. Tree Physiology 24: 651-660.

Norizan, M. S., Mohammad, M. K., Kamaruzaman, A. A. B., \& Arifin, I. 2016. Evaluation of Organic Matter as Potting Media. The Planter, Kuala Lumpur 91: 101-111.

Mounier, E., Pervent, M., Ljung, K., \& Nacry, P. 2014. Auxinmediated nitrate signalling by NRT1.1 participates in the adaptive response of Arabidopsis root architecture to the spatial heterogeneity of nitrate availability. Plant, Cell Enviroment 37: 162-174.

Oliet, J. A., Tejada, M., Salifu, K. F., Collazos, A., \& Jacobs, D. F. 2009. Performance and nutrient dynamics of holm oak (Quercus ilex L.) seedlings in relation to nursery nutrient loading and post-transplant fertility. European Journal of Forest Research 128: 253-263.

Said, F. M., Mariyappan, T., Ramli N. H., \& Hisham, E. 2016. The effect of weight ratio on the physiochemical properties of compost from palm oil mill effluent (POME) sludge and decanter cake. Australian Journal of Basic and Applied Sciences 10: 34-39.

Rivenshield, A., \& Bassuk, N. L. 2007. Using organic amendments to decrease bulk density and increase macroporosity in compacted soils. International Sciety Arboriculture 33: 140-146.

Robbins, J. 2013. Growing media for container production in a greenhouse or nursery.

FSA6097-PD-8-2018RV, from Agriculture and Natural Resources https://www.uaex.edu/publications/PDF/FSA-6097.pdf

Rosenani, A. B., Rovica, R., Cheah, P. M., \& Lim, C. T. $2016 \mathrm{~b}$. Growth performance and nutrient uptake of oil palm seedling in prenursery stage as influenced by oil palm waste compost in growing media. Hindawi Publication Corporation International Journal Agronomy 2016: 1-8.

Schlemmer. M., Gitelson, A., Schepers, J. S., Ferguson, R. B., \& Peng, Y. 2013. Remote estimation of nitrogen and chlorophyll contents in maize at leaf and canopy levels. International Journal of Applied Earth Observations and Geoinformation 25: 47-54.

Shahzad, Z., \& Amtmann, A. 2017. Food for thought: how nutrients regulate root system architecture. Plant Biology 39: $80-87$.

Shen, J., Li, C., Mi, G., Li, L., Yuan, L., Jiang, R., \& Zhang, F. 2013. Maximizing root/rhizosphere efficiency to improve crop productivity and nutrient use efficiency in intensive agriculture of China. Experimental Botany 64: 1181-1192.

Sime Darby Research 2018. Chemical, physical and microbiology. Laboratory Accreditation Scheme of Malaysia 2: 1-17.

Valarini, P. J., Curaqueo, G., Seguel, A., Manzano, K., Rubio, R., Carnejo, P., \& Borie, F. 2009. Effect of compost application on some properties of a volcanic soil from central south Chile. Chilean Journal Agricultural Reserach 69: 416-425.

Woolfolk, M. W., \& Friend, A. L. 2003. Growth response of cottonwood roots to varied NH4:NO3 ratios in enriched patches. Journal of Tree Physiology 23: 427-432.

Xu, J. M., Tang, C., \& Chen, Z. L. 2006. The role of plant residues in $\mathrm{pH}$ change of acid soils differing in initial $\mathrm{pH}$. Soil Biol Biochem 38: 709-719.

Zuraidah., Y. Harun., M. H., \& Zulkifli, Z. 2015. Oil palm roots adaptation under soil compacted by mechanisation. International Journal of Agricultural Science and Research 5: 331342. 